\title{
P01-166
}

\section{RISK OF POSTPARTUM DEPRESSION IN ASSOCIATION WITH SERUM LEPTIN LEVELS: A NESTED CASE-CONTROL} STUDY WITHIN THE UPPSAT COHORT

\author{
A. Skalkidou ${ }^{1}$, I. Sundström-Poromaa' ${ }^{1}$, S. Sylven ${ }^{1}$, M. Olovsson ${ }^{1}$, A. Larsson², F. Papadopoulos ${ }^{3}$ \\ ${ }^{1}$ Department of Obstetrics and Gynecology, Institution of Womens and Childrens Health, ${ }^{2}$ Department of Clinical Chemistry, \\ Akademiska Sjukhuset, ${ }^{3}$ Department of Neurosciences, Upspala University, Uppsala, Sweden
}

\begin{abstract}
Although postpartum depression (PPD) is a common condition, it often goes undiagnosed and untreated, with devastating consequences for the woman's ability to perform daily activities, to bond with her infant and to relate to the infant's father. Leptin, a protein synthesised in the adipose tissue and involved in regulation of food intake and energy expenditure has been related to depressive disorders, but studies report conflicting results. The aim of this study was to evaluate the association between serum leptin levels at the time of delivery and the subsequent development of postpartum depression in women, using data from a population-based cohort of delivering women in Uppsala, Sweden. Three hundred and sixty five women from which serum was obtained at the time of delivery filled out at least one of three pre-coded questionnaires containing the Edinbourgh Scale for Postnatal Depression (EPDS) five days, six weeks and six months after delivery. Crude mean leptin levels did not significantly differ between cases of PPD and controls. Using linear regression analysis and adjusting for maternal age, body-mass index, smoking, interleukin-6 levels, duration of gestation, gender and birth weight of the newborn, the EPDS scores at five days, six weeks and six months after delivery were negatively correlated with leptin levels at delivery $(p<0.05)$. Serum leptin levels at delivery were found to be negatively correlated with self reported depression during the first six months after delivery.
\end{abstract}

